\title{
Spherical Symmetric Solitons of Interacting Spinor, Scalar and Gravitational Fields in General Relativity
}

\author{
Jonas Edou', Alain Adomou ${ }^{1,2}$, Siaka Massou ${ }^{1}$ \\ ${ }^{1}$ Department of Theoretical Physics and Mathematics, Laboratory of Physics Mathematics and Theoretical Physics, \\ University of Abomey-Calavi, Abomey-Calavi, Benin \\ ${ }^{2}$ National Higher Institute of Industrial Technology, INSTI-Lokossa, National University of Sciences, Technology, \\ Engineering and Mathematics of Abomey, Abomey, Benin \\ Email: edjonas131988@gmail.com
}

How to cite this paper: Edou, J., Adomou, A. and Massou, S. (2021) Spherical Symmetric Solitons of Interacting Spinor, Scalar and Gravitational Fields in General Relativity. Journal of High Energy Physics, Gravitation and Cosmology, 7, 52-65. https://doi.org/10.4236/jhepgc.2021.71002

Received: September 27, 2020

Accepted: November 28, 2020

Published: December 1, 2020

Copyright (c) 2021 by author(s) and Scientific Research Publishing Inc. This work is licensed under the Creative Commons Attribution International License (CC BY 4.0).

http://creativecommons.org/licenses/by/4.0/

\begin{abstract}
The concept of soliton as regular localized stable solutions of nonlinear differential equations is being widely utilized in pure science for various aims. In present analysis, the soliton concept is used as a model in order to describe the configurations of elementary particles in general relativity. To this end, our study deals with the spherical symmetric solitons of interacting Spinor, Scalar and Gravitational Fields in General Relativity. Thus, exact spherical symmetric general solutions to the interaction of spinor, scalar and gravitational field equations have been obtained. The Einstein equations have been transformed into a Liouville equation type and solved. Let us emphasize that these solutions are regular with localized energy density and finite total energy. In addition, the total charge and spin are limited. Moreover, the obtained solutions are soliton-like solutions. These solutions can be used in order to describe the configurations of elementary particles.
\end{abstract}

\section{Keywords}

Liouville Type Equation, Elementary Particles, Einstein's Equations, Metric Functions

\section{Introduction}

The theory of solitons in general relativity was first elaborated by G. N. Shikin in 1995. He formulated the requirements to be fulfilled by solitons [1]. His research work allowed an intensive study on soliton in general relativity by many authors. In a series of papers, exact plane-symmetric solutions to the spinor and gravita- 
tional field equations have been obtained. The role of gravitational field and the nonlinear terms in the formation of the field configurations with limited total energy, spin and charge have been thoroughly investigated. Let us emphasize that the total charge and spin of the self-consistent system of spinor and gravitational field equations are unlimited. The divergence of the total charge and spin is related to the non-consideration of the torsion and the properties of the static plane-symmetric utilized [2] [3] [4]. For an excellent review of the interacting scalar and spinor fields in plane-symmetric metric refer to [5] [6] [7]. Note that in [5] [6] [7] the obtained solutions are exact regular with localized energy density and finite total energy. Nevertheless, the total charge and spin are not bounded. The unlimited problem of the charge and the spin is resolved in a series of interesting articles [8]-[13]. The gravitational field is given by a spherically symmetric metric.

The aim of the paper was to study the role of the interaction of nonlinear spinor, scalar and gravitational fields in the formation of configurations with localized energy density and limited energy, spin and charge of the spinor field.

The paper is organized as follows. Section 2 deals with general relativistic equations. The lagrangian of the self-consistent interaction spinor, scalar and gravitational fields has been defined. The gravitational field in our study is given by a spherically symmetric metric. Section 3 addresses the main results. The fundamental field equations have been solved and their localisation properties have been examined. Section 4 deals with the discussion of the main results. Thus, we studied in detail the spinor, scalar and gravitational interaction equations by choosing the concrete form of the arbitrary function $F(S)$ under the form $F(S)=\frac{\lambda S^{n}}{1-\lambda S^{n}}$. Finally, concluding remarks and future work are outlined in Section 5 .

\section{General Relativistic Equations}

The lagrangian of the self-consistent system of interaction between spinor, scalar and gravitational fields may be written under the following form [5]:

$$
\begin{aligned}
L & =L_{g}+L_{S_{p}}+L_{S_{c}}+L_{i n t} \\
& =\frac{R}{2 \chi}+\frac{i}{2}\left(\bar{\psi} \gamma^{\mu} \nabla_{\mu} \psi-\nabla_{\mu} \bar{\psi} \gamma^{\mu} \psi\right)-m \bar{\psi} \psi+\frac{1}{2} \varphi_{, \mu} \varphi^{, \mu}+\frac{1}{2} \varphi_{, \mu}{ }^{, \mu} F(S) .
\end{aligned}
$$

$L_{g} ; L_{S_{p}} ; L_{S_{c}}$ and $L_{\text {int }}$ correspond respectively to gravitational field lagrangian, free spinor field lagrangian, free scalar field lagrangian and interaction lagrangian. They are defined as follows:

$$
\begin{gathered}
L_{g}=\frac{R}{2 \chi}, \\
L_{S_{p}}=\frac{i}{2}\left(\bar{\psi}^{\mu} \nabla_{\mu} \psi-\nabla_{\mu} \bar{\psi} \gamma^{\mu} \psi\right)-m \bar{\psi} \psi \\
L_{S_{c}}=\frac{1}{2} \varphi_{, \mu} \varphi^{\mu}
\end{gathered}
$$




$$
L_{i n t}=\frac{1}{2} \varphi_{, \mu} \varphi^{, \mu} F(S)
$$

$R$ denotes the scalar curvature; $\kappa=\frac{8 \pi G}{c^{4}}$ is Einstein's gravitational constant; $G$ is Newton's universal gravitational constant; $c$ is the velocity of light; $F(S)$ is an arbitrary function of the invariant $S=\bar{\psi} \psi$.

In the sequel, in ordor to simplify the expressions, we shall consider:

$$
\begin{gathered}
L_{m}=L_{S_{p}}+L_{S_{c}}+L_{i n t} \\
\phi(S)=1+F(S) \Rightarrow \phi^{\prime}(S)=\frac{\mathrm{d} \phi(S)}{\mathrm{d} S}=\frac{\mathrm{d} F(S)}{\mathrm{d} S}
\end{gathered}
$$

The grvitational field in our case is given by a spherical symmetric space-time via the metric which is defined under the following form:

$$
\mathrm{d} s^{2}=\mathrm{e}^{2 \gamma} \mathrm{d} t^{2}-\mathrm{e}^{2 \alpha} \mathrm{d} \xi^{2}-\mathrm{e}^{2 \beta}\left[\mathrm{d} \theta^{2}+\sin ^{2}(\theta) \mathrm{d} \varphi^{2}\right] .
$$

For simplicity reason, the speed of light has been taken to be unity $(c=1)$. The metric functions $\alpha, \beta$ and $\gamma$ are time and angular coordinates $\theta$ and $\varphi$ independent. They are functions of spatial variable $\xi$ alone which is defined as in [8] in the form $\xi=\frac{1}{r}$, where $r$ stands for the radial component of the spherical symmetric metric. These metric functions obey the harmonic coordinates conditions:

$$
\alpha=2 \beta+\gamma
$$

Variation of (1) with respect to the spinor field $\psi$ and its conjugate $\bar{\psi}$, we establish the nonlinear spinor field equations as follows:

$$
\begin{aligned}
& i \gamma^{\mu} \nabla_{\mu} \psi-m \psi+\frac{1}{2} \varphi_{, \mu} \varphi^{\mu} \phi^{\prime}(S) \psi=0 \\
& i \nabla_{\mu} \bar{\psi} \gamma^{\mu}+m \bar{\psi}+\frac{1}{2} \varphi_{, \mu} \varphi^{, \mu} \phi^{\prime}(S) \bar{\psi}=0
\end{aligned}
$$

Similarly, varying of (1) with respect to the scalar field we obtain the following scalar field equation:

$$
\frac{1}{\sqrt{-g}} \frac{\partial}{\partial \xi^{v}}\left[\sqrt{-g} g^{\mu v} \varphi_{, \mu} \phi(S)\right]=0
$$

Then, the general form of Einstein's field equation is:

$$
G_{\mu}^{v}=R_{\mu}^{v}-\frac{1}{2} \delta_{\mu}^{v} R=-\chi T_{\mu}^{v},
$$

In virtue of (8) and (9), expression (13) becomes:

$$
\begin{gathered}
G_{0}^{0}=\mathrm{e}^{-2 \alpha}\left(2 \beta^{\prime \prime}-2 \gamma^{\prime} \beta^{\prime}-\beta^{\prime 2}\right)-\mathrm{e}^{-2 \beta}=-\chi T_{0}^{0}, \\
G_{1}^{1}=\mathrm{e}^{-2 \alpha}\left(2 \beta^{\prime} \gamma^{\prime}+\beta^{\prime 2}\right)-\mathrm{e}^{-2 \beta}=-\chi T_{1}^{1}, \\
G_{2}^{2}=\mathrm{e}^{-2 \alpha}\left(\beta^{\prime \prime}+\gamma^{\prime \prime}-2 \beta^{\prime} \gamma^{\prime}-\beta^{\prime 2}\right)=-\chi T_{2}^{2}, \\
G_{2}^{2}=G_{3}^{3}, \quad T_{2}^{2}=T_{3}^{3},
\end{gathered}
$$


where prime (') denotes differentiation with respect to $\xi, T_{\mu}^{v}$ is the energy momentum tensor of the spinor, scalar fields and its interaction, $G_{\mu}^{v}$ is Einstein's tensor, $R_{\mu}^{v}$ is Ricci's tensor and $\delta_{\mu}^{v}$ is Kronecker's symbol which is 0 if $\mu \neq v$ and 1 if $\mu=v$.

The metric energy-momentum tensor of the interaction of the spinor and scalar fields field can be written as follows:

$$
T_{\mu}^{v}=T_{s_{p \mu}}^{v}+T_{s_{c \mu}}^{v}+T_{i n t \mu}^{v},
$$

otherwise

$$
T_{\mu}^{v}=\frac{i}{4} g^{\nu \rho}\left(\bar{\psi} \gamma_{\mu} \nabla_{\nu} \psi+\bar{\psi} \gamma_{\nu} \nabla_{\mu} \psi-\nabla_{\mu} \bar{\psi} \gamma_{\nu} \psi-\nabla_{\nu} \bar{\psi} \gamma_{\mu} \psi\right)+\varphi_{, \mu} \varphi_{, \nu} \phi(S)-\delta_{\mu}^{v} L_{m}
$$

Taking into account (10) and (11), we rewrite $L_{m}$ under the form:

$$
\begin{aligned}
L_{m} & =-\frac{1}{2}\left(\bar{\psi} \frac{\partial L_{i n t}}{\partial \bar{\psi}}+\frac{\partial L_{i n t}}{\partial \psi} \psi\right)+L_{i n t}+L_{s c} \\
& =-S L_{i n t}^{\prime}+L_{i n t}+L_{s c}
\end{aligned}
$$

Taking into account (19) and (21), let us try to rewrite explicitly the non null components of the metric energy-momentum tensor $T_{\mu}^{v}$. In this optic, we obtain:

$$
\begin{gathered}
T_{0}^{0}=T_{2}^{2}=T_{3}^{3}=\frac{1}{2}\left(\varphi^{\prime}\right)^{2}\left[\phi(S)-\phi^{\prime}(S) S\right] \cdot \mathrm{e}^{-2 \alpha(\xi)} \\
T_{1}^{1}=\frac{i}{2}\left(\bar{\psi} \gamma^{1} \nabla_{1} \psi-\nabla_{1} \bar{\psi}^{1} \psi\right)+\frac{1}{2}\left(\varphi^{\prime}\right)^{2}\left[\phi(S)-\phi^{\prime}(S) S\right] \cdot \mathrm{e}^{-2 \alpha(\xi)}
\end{gathered}
$$

In expressions (10), (11) and (19), $\nabla_{\mu}$ is the derivative covariant of spinor. It is connected to the spinor affine connection matrices $\Gamma_{\mu}(\xi)$ [14] [15]:

$$
\nabla_{\mu} \psi=\frac{\partial \psi}{\partial \xi^{\mu}}-\Gamma_{\mu} \psi \quad \text { or } \quad \nabla_{\mu} \bar{\psi}=\frac{\partial \bar{\psi}}{\partial \xi^{\mu}}+\Gamma_{\mu} \bar{\psi} .
$$

In the above equations, $\gamma^{\mu}$ are Dirac's matrices in curved space-time. In order to define $\gamma^{\mu}$, let us use the equalities:

$$
\begin{gathered}
g_{\mu \nu}(\xi)=e_{\mu}^{a}(\xi) e_{\nu}^{b}(\xi) \eta_{a b} \\
\gamma_{\mu}(\xi)=e_{\mu}^{a}(\xi) \bar{\gamma}_{a},
\end{gathered}
$$

where $\eta_{a b}=\operatorname{diag}(1,-1,-1,-1)$ is the metric of Minkowski and $e_{\mu}^{a}(\xi)$ are tetradic 4 -vectors.

With the relation (25), we have:

$$
\gamma^{0}(\xi)=\mathrm{e}^{-\gamma} \bar{\gamma}^{0}, \gamma^{1}(\xi)=\mathrm{e}^{-\alpha} \bar{\gamma}^{1}, \gamma^{2}(\xi)=\mathrm{e}^{-\beta} \bar{\gamma}^{2}, \gamma^{3}(\xi)=\frac{\mathrm{e}^{-\beta} \bar{\gamma}^{3}}{\sin \theta}, \gamma^{5}(\xi)=\bar{\gamma}^{5}
$$

For the matrices $\bar{\gamma}^{a}$ in flat space-time, we take [16]:

$$
\bar{\gamma}^{0}=\left(\begin{array}{cccc}
1 & 0 & 0 & 0 \\
0 & 1 & 0 & 0 \\
0 & 0 & -1 & 0 \\
0 & 0 & 0 & -1
\end{array}\right) ; \quad \bar{\gamma}^{1}=\left(\begin{array}{cccc}
0 & 0 & 0 & 1 \\
0 & 0 & 1 & 0 \\
0 & -1 & 0 & 0 \\
-1 & 0 & 0 & 0
\end{array}\right)
$$




$$
\begin{gathered}
\bar{\gamma}^{2}=\left(\begin{array}{cccc}
0 & 0 & 0 & -i \\
0 & 0 & i & 0 \\
0 & i & 0 & 0 \\
-i & 0 & 0 & 0
\end{array}\right) ; \quad \bar{\gamma}^{3}=\left(\begin{array}{cccc}
0 & 0 & 1 & 0 \\
0 & 0 & 0 & -1 \\
-1 & 0 & 0 & 0 \\
0 & 1 & 0 & 0
\end{array}\right) \\
\gamma^{5}=\bar{\gamma}^{5}=\left(\begin{array}{cccc}
0 & 0 & -1 & 0 \\
0 & 0 & 0 & -1 \\
-1 & 0 & 0 & 0 \\
0 & -1 & 0 & 0
\end{array}\right)
\end{gathered}
$$

At present, let us define the affine connection matrices of the spinor. To this end, the general form of $\Gamma_{\mu}(\xi)$ is:

$$
\Gamma_{\mu}(\xi)=\frac{1}{4} g_{\rho \mu}\left(\partial_{\mu} e_{\sigma}^{b} e_{a}^{\rho}-\Gamma_{\mu \sigma}^{\rho}\right) \gamma^{\delta} \gamma^{\sigma},
$$

In (27), $\Gamma_{\mu \sigma}^{\rho}$ are Christoffel's symbols. From expression (27), we have the components of the affine connection matrices of the spinor in the following way:

$$
\begin{aligned}
& \Gamma_{0}=-\frac{1}{2} \mathrm{e}^{-2 \beta} \bar{\gamma}^{0} \bar{\gamma}^{1} \gamma^{\prime}, \quad \Gamma_{1}=0, \quad \Gamma_{2}=\frac{1}{2} \mathrm{e}^{-\beta-\gamma} \bar{\gamma}^{2} \bar{\gamma}^{1} \beta^{\prime}, \\
& \Gamma_{3}=\frac{1}{2}\left(\mathrm{e}^{-\beta-\gamma} \bar{\gamma}^{3} \bar{\gamma}^{1} \beta^{\prime} \sin \theta+\bar{\gamma}^{3} \bar{\gamma}^{2} \cos \theta\right) .
\end{aligned}
$$

Equation (12) has solution:

$$
\varphi^{\prime}(\xi) \phi(S)=C \Rightarrow \varphi^{\prime}(\xi)=\frac{C}{\phi(S)}, \quad C=\text { cste. }
$$

According to Einstein's convention of sommation, we get:

$$
\gamma^{\mu} \Gamma_{\mu}=-\frac{1}{2}\left(\mathrm{e}^{-\alpha} \alpha^{\prime} \bar{\gamma}^{1}+\bar{\gamma}^{2} \mathrm{e}^{-\beta} \cot \theta\right) .
$$

Then, using expressions (24), (29) and (30), we can rewrite Equations (10) and (11) as follows:

$$
\begin{aligned}
& i e^{-\alpha} \bar{\gamma}^{1}\left(\partial_{\xi}+\frac{1}{2} \alpha^{\prime}\right) \psi+\frac{i}{2} \bar{\gamma}^{2} \mathrm{e}^{-\beta} \psi \cot \theta-m \psi-\frac{C^{2}}{2} \mathrm{e}^{-2 \alpha} \frac{\phi^{\prime}(S)}{\phi^{2}(S)} \psi=0, \\
& \mathrm{ie}^{-\alpha} \bar{\gamma}^{1}\left(\partial_{\xi}+\frac{1}{2} \alpha^{\prime}\right) \bar{\psi}+\frac{i}{2} \bar{\gamma}^{2} \mathrm{e}^{-\beta} \bar{\psi} \cot \theta+m \bar{\psi}+\frac{C^{2}}{2} \mathrm{e}^{-2 \alpha} \frac{\phi^{\prime}(S)}{\phi^{2}(S)} \bar{\psi}=0 .
\end{aligned}
$$

Further setting $\psi(\xi)=V_{\delta}(\xi)$ with $\delta=1,2,3,4$, from (31), we obtain the following set of equations:

$$
\begin{aligned}
& V_{4}^{\prime}+\frac{1}{2} \alpha^{\prime} V_{4}-\frac{i}{2} \mathrm{e}^{\alpha-\beta} V_{4} \cot \theta+i m \mathrm{e}^{\alpha} V_{1}-i \frac{C^{2}}{2} Q^{\prime}(S) \mathrm{e}^{-\alpha} V_{1}=0, \\
& V_{3}^{\prime}+\frac{1}{2} \alpha^{\prime} V_{3}+\frac{i}{2} \mathrm{e}^{\alpha-\beta} V_{3} \cot \theta+i m \mathrm{e}^{\alpha} V_{2}-i \frac{C^{2}}{2} Q^{\prime}(S) \mathrm{e}^{-\alpha} V_{2}=0, \\
& V_{2}^{\prime}+\frac{1}{2} \alpha^{\prime} V_{2}-\frac{i}{2} \mathrm{e}^{\alpha-\beta} V_{2} \cot \theta-i m \mathrm{e}^{\alpha} V_{3}+i \frac{C^{2}}{2} Q^{\prime}(S) \mathrm{e}^{-\alpha} V_{3}=0, \\
& V_{1}^{\prime}+\frac{1}{2} \alpha^{\prime} V_{1}+\frac{i}{2} \mathrm{e}^{\alpha-\beta} V_{1} \cot \theta-i m \mathrm{e}^{\alpha} V_{4}+i \frac{C^{2}}{2} Q^{\prime}(S) \mathrm{e}^{-\alpha} V_{4}=0,
\end{aligned}
$$

where function $Q(S)$ is: 


$$
Q(S)=\frac{1}{\phi(S)}
$$

The functions $V_{1}, V_{2}, V_{3}$ and $V_{4}$ are connected by the relation:

$$
V_{1}^{2}-V_{2}^{2}-V_{3}^{2}+V_{4}^{2}=\text { cste. }
$$

The following section deals with the main results.

\section{Main Results}

Summing the set of Equations (33)-(36), we infer that the invariant function:

$$
S=\bar{\psi} \psi=V_{1} * V_{1}+V_{2} * V_{2}-V_{3} * V_{3}-V_{4} * V_{4},
$$

satisfies a firt order differential equation as follows:

$$
\frac{\mathrm{d} S}{\mathrm{~d} \xi}+\alpha^{\prime}(\xi) S=0
$$

Equation (40) has solution:

$$
S(\xi)=C_{1} \exp [-\alpha(\xi)], \quad C_{1}=\text { const. }
$$

Expression (41), reflects the natural link between the nonlinear spinor field of elementary particles and their own gravitational field.

By Considering the spinor field equation in the form (31) and the conjugate one in the form (32), from (23), the momentum $T_{1}^{1}$ may be rewritten in the following form:

$$
T_{1}^{1}=m S+\frac{1}{2}(\varphi)^{2} \phi(S) \mathrm{e}^{-2 \alpha}=m S+\frac{C^{2}}{2} Q(S) \mathrm{e}^{-2 \alpha}
$$

In the following paragraph, we shall solve Einstein's field equations in order to determine the general expressions of the metric functions $\alpha, \beta$ and $\gamma$ and then the link which exits between them.

In this perspective, in virtue of $T_{0}^{0}=T_{2}^{2}$, substraction of Einstein's Equations (14) and (16) leads to the following equation:

$$
\beta^{\prime \prime}-\gamma^{\prime \prime}=\mathrm{e}^{2 \beta+2 \gamma} \text {. }
$$

The previous equation can be transformed into a Liouville equation type to produce the following solutions (refer to [1], page 30):

$$
\begin{gathered}
\beta(\xi)=\frac{A}{4}\left(1+\frac{2}{B}\right) \ln \left[\frac{A}{B T^{2}\left(h, \xi+\xi_{1}\right)}\right]=\left(1+\frac{2}{B}\right) \gamma(\xi), \\
\gamma(\xi)=\frac{A}{4} \ln \left[\frac{A}{B T^{2}\left(h, \xi+\xi_{1}\right)}\right],
\end{gathered}
$$

$A$ and $D$ are integration constants. $T$ is a function. It is defined under the form:

$$
T\left(h, \xi+\xi_{1}\right)=\left\{\begin{array}{l}
\frac{1}{h} \sinh \left[h\left(\xi+\xi_{1}\right)\right], h>0 \\
\left(\xi+\xi_{1}\right), h=0 \\
\frac{1}{h} \sin \left[h\left(\xi+\xi_{1}\right)\right], h<0
\end{array}\right.
$$


where $h$ and $\xi_{1}$ are integration constants.

From (44), (45) and (9), one finds the explicit form of the metric function $\alpha(\xi)$ as follows:

$$
\alpha(\xi)=\frac{A}{2}\left(\frac{3}{2}+\frac{2}{B}\right) \ln \left[\frac{A}{B T^{2}\left(h, \xi+\xi_{1}\right)}\right] .
$$

Then, the metric functions $\alpha(\xi), \beta(\xi)$ and $\gamma(\xi)$ are connected as follows:

$$
\beta(\xi)=\frac{2+B}{4+3 B} \alpha(\xi) ; \quad \gamma(\xi)=\frac{B}{4+3 B} \alpha(\xi) .
$$

Let us note that Einstein's Equation (15) is a first integral of Equations (14) and (16). It is also a first order differential equation. By substituting, (48) and (42) into (15), we obtain:

$$
\left(\alpha^{\prime}\right)^{2}=\frac{(4+3 B)^{2}}{3 B^{2}+8 B+4} \mathrm{e}^{2 \alpha}\left[\mathrm{e}^{-\frac{4+2 B}{4+3 B} \alpha}-\chi\left(m S+\frac{C^{2}}{2} Q(S) \mathrm{e}^{-2 \alpha}\right)\right]
$$

Substituting $\alpha^{\prime}=-\frac{1}{S} \frac{\mathrm{d} S}{\mathrm{~d} \xi}$ and $S(\xi)=C_{1} \mathrm{e}^{-\alpha(\xi)}$, into (49), we have:

$$
\frac{\mathrm{d} S}{\mathrm{~d} \xi}= \pm \frac{4+3 B}{\sqrt{3 B^{2}+8 B+4}} \sqrt{\left[\left(\frac{S^{2}}{C_{1}^{2}}\right)^{\frac{2+B}{4+3 B}}-\chi\left(m S+\frac{C^{2}}{2 C_{1}^{2}} S^{2} Q(S)\right)\right]}
$$

Equation (50) has the general analytical solutions under the form:

$$
\int \frac{\mathrm{d} S}{\frac{4+3 B}{\sqrt{3 B^{2}+8 B+4}} \sqrt{\left[\left(\frac{S^{2}}{C_{1}^{2}}\right)^{\frac{2+B}{4+3 B}}-\chi\left(m S+\frac{C^{2}}{2 C_{1}^{2}} S^{2} Q(S)\right)\right]}}= \pm\left(\xi+\xi_{0}\right)
$$

Setting a concrete form of the function $F(S)$, from (7) we can find $\phi(S)$. Knowing $\phi(S)$, we can determine $Q(S)$, from (37). Thus, from (51), the invariant function $S=\bar{\psi} \psi$ can be found. Then, if $S(\xi)$ is known, we can determine $\alpha(\xi)$ from (41). Finally, the metric functions $\beta(\xi)$ and $\gamma(\xi)$ can be determined from expression (48).

Considering the concrete expression of the invariant function $S(\xi)=C_{1} \mathrm{e}^{-\alpha(\xi)}$, we can establish the regularity properties of the solutions obtained. Studying the distribution of the energy per unit invariant volume $T_{0}^{0} \sqrt{-3_{g}}$, we can establish the localization properties of the solutions.

In the sequel, we shall get a concrete form of the functions $V_{\delta}(\xi)$ by solving Equations (33)-(36) in a more compact form if we pass to the functions $U_{\delta}(\xi)=\mathrm{e}^{-\frac{\alpha}{2}} V_{\delta}(\xi)$, with $\delta=1,2,3,4$. To this end, introducing the new functions $U_{\delta}(\xi)$ into (33)-(36), we obtain the following set of equations:

$$
U_{4}^{\prime}(\xi)-\frac{i}{2}\left(\frac{C_{1}}{S}\right)^{\frac{2+2 B}{4+3 B}} U_{4}(\xi) \cot \theta+i\left[\frac{m C_{1}}{S}-\frac{C^{2}}{C_{1}} S Q^{\prime}(S)\right] U_{1}(\xi),
$$




$$
\begin{aligned}
& U_{3}^{\prime}(\xi)+\frac{i}{2}\left(\frac{C_{1}}{S}\right)^{\frac{2+2 B}{4+3 B}} U_{3}(\xi) \cot \theta+i\left[\frac{m C_{1}}{S}-\frac{C^{2}}{C_{1}} S Q^{\prime}(S)\right] U_{2}(\xi), \\
& U_{2}^{\prime}(\xi)-\frac{i}{2}\left(\frac{C_{1}}{S}\right)^{\frac{2+2 B}{4+3 B}} U_{2}(\xi) \cot \theta-i\left[\frac{m C_{1}}{S}-\frac{C^{2}}{C_{1}} S Q^{\prime}(S)\right] U_{3}(\xi), \\
& U_{1}^{\prime}(\xi)+\frac{i}{2}\left(\frac{C_{1}}{S}\right)^{\frac{2+2 B}{4+3 B}} U_{1}(\xi) \cot \theta-i\left[\frac{m C_{1}}{S}-\frac{C^{2}}{C_{1}} S Q^{\prime}(S)\right] U_{4}(\xi),
\end{aligned}
$$

where:

$$
U_{\delta}^{\prime}(\xi)=\left[V_{\delta}^{\prime}(\xi)+\frac{1}{2} \alpha^{\prime}(\xi) V_{\delta}(\xi)\right] \exp \left[\frac{1}{2} \alpha(\xi)\right]
$$

Let us re-express Equations (52)-(55) to the function of argument $S(\xi)$, i.e. $W_{\delta}(S)=U_{\delta}(\xi), S(\xi)=C_{1} \exp [-\alpha(\xi)]$. In these conditions, we have the following set of equations for the functions $W_{\delta}(S)$ :

$$
\begin{aligned}
& \frac{\mathrm{d} W_{4}}{\mathrm{~d} S}-i B(S) W_{4}-M(S) W_{1}=0 \\
& \frac{\mathrm{d} W_{3}}{\mathrm{~d} S}+i B(S) W_{3}-M(S) W_{2}=0 \\
& \frac{\mathrm{d} W_{2}}{\mathrm{~d} S}-i B(S) W_{2}+M(S) W_{3}=0, \\
& \frac{\mathrm{d} W_{1}}{\mathrm{~d} S}+i B(S) W_{1}+M(S) W_{4}=0
\end{aligned}
$$

with

$$
B(S)=\frac{1}{2} \frac{\left(\frac{C_{1}}{S}\right)^{\frac{2+2 B}{4+3 B}} \cot \theta}{\sqrt{\left[\left(\frac{S^{2}}{C_{1}^{2}}\right)^{\frac{2+B}{4+3 B}}-\chi\left(m S+\frac{C^{2}}{2 C_{1}^{2}} S^{2} Q(S)\right)\right]}},
$$

and

$$
M(S)=\frac{1}{2} \frac{\frac{m C_{1}}{S}-\frac{C^{2} S}{2 C_{1}} Q^{\prime}(S)}{\sqrt{\left[\left(\frac{S^{2}}{C_{1}^{2}}\right)^{\frac{2+B}{4+3 B}}-\chi\left(m S+\frac{C^{2}}{2 C_{1}^{2}} S^{2} Q(S)\right)\right]}} .
$$

Let us pass from the set of first-order differential Equation (57)-(60) to a set of second-order differential equations. By doing so, differentiating Equation (57) in $S$ and introducing into the result the expressions of the function $W_{1}(S)$ and $W_{1}^{\prime}(S)$, we obtain a second-order differential equation for the function $W_{4}(S)$ as follows: 


$$
\begin{aligned}
& W_{4}^{\prime \prime}(S)-\frac{M^{\prime}(S)}{M(S)} W_{4}^{\prime}(S) \\
& +\left[B^{2}(S)-M^{2}(S)+i \frac{B(S) M^{\prime}(S)-M(S) B^{\prime}(S)}{M(S)}\right] W_{4}(S)=0
\end{aligned}
$$

Similarly differentiating Equation (60) and introducing into the result the expression of $W_{4}(S)$ and the expression of its derivative, we obtain the second-order differential equation for the function $W_{1}(S)$ :

$$
\begin{aligned}
& W_{1}^{\prime \prime}(S)-\frac{M^{\prime}(S)}{M(S)} W_{1}^{\prime}(S) \\
& +\left[B^{2}(S)-M^{2}(S)+i \frac{M(S) B^{\prime}(S)-B(S) M^{\prime}(S)}{M(S)}\right] W_{1}(S)=0
\end{aligned}
$$

Summing (63)-(64) and setting $X(S)=W_{1}(S)+W_{4}(S)$, we obtain the following second-order differential equations of the function $X(S)$ :

$$
X^{\prime \prime}(S)-\frac{M^{\prime}(S)}{M(S)} X^{\prime}(S)+2\left[B^{2}(S)-M^{2}(S)\right] X(S)=0
$$

Under the condition $B^{2}(S)=(1-\varepsilon) M^{2}(S)$ with $0<\varepsilon \leq 1$ [8], the solution of Equation (65) is:

$$
W_{1}+W_{4}=a_{0} \sinh N_{1}(S), \quad a_{0}=\text { const },
$$

where

$$
N_{1}(S)=\sqrt{2 \varepsilon} \int M(S) \mathrm{d} S+R_{1}, \quad R_{1}=\text { const } .
$$

Substracting Equations (57) and (60) and taking into account (66), we get:

$$
W_{1}-W_{4}=-i a_{0}\left(\frac{\sqrt{1-\varepsilon}-1}{\sqrt{2 \varepsilon}}\right) \cosh N_{1}(S)
$$

From expressions (66) and (68), we define the functions $W_{1}$ and $W_{4}$ as follows:

$$
\begin{aligned}
& W_{1}(S)=\alpha_{0}\left[\sinh N_{1}(S)-i\left(\frac{\sqrt{1-\varepsilon}-1}{\sqrt{2 \varepsilon}}\right) \cosh N_{1}(S)\right] \\
& W_{4}(S)=\alpha_{0}\left[\sinh N_{1}(S)+i\left(\frac{\sqrt{1-\varepsilon}-1}{\sqrt{2 \varepsilon}}\right) \cosh N_{1}(S)\right]
\end{aligned}
$$

The same operating on Equations (58) and (59) leads to the following expressions:

$$
\begin{aligned}
& W_{2}(S)=D_{0}\left[\cosh N_{2}(S)+i\left(\frac{\sqrt{1-\varepsilon}+1}{\sqrt{2 \varepsilon}}\right) \sinh N_{2}(S)\right], \\
& W_{3}(S)=D_{0}\left[\cosh N_{2}(S)-i\left(\frac{\sqrt{1-\varepsilon}+1}{\sqrt{2 \varepsilon}}\right) \sinh N_{2}(S)\right],
\end{aligned}
$$

with

$$
N_{2}(S)=\sqrt{2 \varepsilon} \int M(S) \mathrm{d} S+R_{2}, \quad R_{2}=\text { const. }
$$


It follows that, the concrete form of the functions $V_{\delta}(\xi)$ is:

$$
\begin{aligned}
V_{1}(S)= & \alpha_{0}\left[\sinh N_{1}(S)-i\left(\frac{\sqrt{1-\varepsilon}-1}{\sqrt{2 \varepsilon}}\right) \cosh N_{1}(S)\right] \\
& \times \exp \left\{-\frac{A}{4}\left(\frac{3}{2}+\frac{2}{B}\right) \ln \left[\frac{A}{B T^{2}\left(h, \xi+\xi_{1}\right)}\right]\right\} \\
V_{2}(S)= & D_{0}\left[\cosh N_{2}(S)+i\left(\frac{\sqrt{1-\varepsilon}+1}{\sqrt{2 \varepsilon}}\right) \sinh N_{2}(S)\right] \\
& \times \exp \left\{-\frac{A}{4}\left(\frac{3}{2}+\frac{2}{B}\right) \ln \left[\frac{A}{B T^{2}\left(h, \xi+\xi_{1}\right)}\right]\right\}, \\
V_{3}(S)= & D_{0}\left[\cosh N_{2}(S)-i\left(\frac{\sqrt{1-\varepsilon}+1}{\sqrt{2 \varepsilon}}\right) \sinh N_{2}(S)\right] \\
& \times \exp \left\{-\frac{A}{4}\left(\frac{3}{2}+\frac{2}{B}\right) \ln \left[\frac{A}{B T^{2}\left(h, \xi+\xi_{1}\right)}\right]\right\}, \\
V_{4}(S)= & \alpha_{0}\left[\sinh N_{1}(S)+i\left(\frac{\sqrt{1-\varepsilon}-1}{\sqrt{2 \varepsilon}}\right) \cosh N_{1}(S)\right] \\
& \times \exp \left\{-\frac{A}{4}\left(\frac{3}{2}+\frac{2}{B}\right) \ln \left[\frac{A}{B T^{2}\left(h, \xi+\xi_{1}\right)}\right]\right\}
\end{aligned}
$$

The exact functions $V_{\delta}(\xi)$ are regular and bounded.

In the sequel, we shall use the concrete form of the functions $V_{\delta}(\xi)$ in order to determine the total charge and total spin.

The general form of the spinor current vector is:

$$
j^{\mu}=\bar{\psi} \gamma^{\mu} \psi
$$

From (78), we deduce the components of the spinor current vector as follows:

$$
\begin{aligned}
& j^{0}= 2 \mathrm{e}^{-\alpha-\gamma}\left\{\alpha_{0}^{2}\left[\sinh ^{2} N_{1}(\xi)+\left(\frac{-1+\sqrt{1-\varepsilon}}{\sqrt{2 \varepsilon}}\right)^{2} \cosh ^{2} N_{1}(\xi)\right]\right. \\
&\left.+D_{0}^{2}\left[\cosh ^{2} N_{2}(\xi)+\left(\frac{-1+\sqrt{1-\varepsilon}}{\sqrt{2 \varepsilon}}\right)^{2} \sinh ^{2} N_{2}(\xi)\right]\right\} \\
& j^{1}=2 \mathrm{e}^{-2 \alpha}\left\{\alpha_{0}^{2}\left[\sinh ^{2} N_{1}(\xi)-\left(\frac{-1+\sqrt{1-\varepsilon}}{\sqrt{2 \varepsilon}}\right)^{2} \cosh ^{2} N_{1}(\xi)\right]\right. \\
&+\left.D_{0}^{2}\left[\cosh ^{2} N_{2}(\xi)-\left(\frac{-1+\sqrt{1-\varepsilon}}{\sqrt{2 \varepsilon}}\right)^{2} \sinh ^{2} N_{2}(\xi)\right]\right\} \\
& j^{2}= 4 \mathrm{e}^{-\alpha-\beta}\left[\alpha_{0}^{2}\left(\frac{-1+\sqrt{1-\varepsilon}}{\sqrt{2 \varepsilon}}\right) \cosh N_{1}(\xi) \sinh N_{1}(\xi)\right. \\
&\left.-D_{0}^{2}\left(\frac{-1+\sqrt{1-\varepsilon}}{\sqrt{2 \varepsilon}}\right) \cosh N_{2}(\xi) \sinh N_{2}(\xi)\right]
\end{aligned}
$$




$$
j^{3}=0
$$

In this study, the configuration is assumed static. Thus, only the component $j^{0}$ is non null. With this assumption, we obtain: $\alpha_{0}=D_{0} ; \varepsilon=1$; $N_{1}(S)=N_{2}(S)=N(S)$. In addition to this, we have $R_{1}=R_{2}=R$.

The component $j^{0}$ determines the charge density of the spinor field and scalar field in interaction:

$$
q(\xi)=\left(j_{0} j^{0}\right)^{\frac{1}{2}}=3 a^{2} \cosh [2 \sigma(x)] \mathrm{e}^{-\alpha} .
$$

The charge density is continuous localized function.

The total charge of the interaction system of spinor and scalar fields is:

$$
Q=\int_{0}^{\xi_{c}} q(\xi) \mathrm{d} \xi .
$$

In the integral (84), $\xi_{c}$ denotes the center of the fields configurations.

The spin tensor of the spinor field reads:

$$
S^{\mu v, \lambda}=\frac{1}{4} \bar{\psi}\left\{\gamma^{\lambda} \sigma^{\mu v}+\sigma^{\mu v} \gamma^{\lambda}\right\} \psi .
$$

The analytical expression $S^{i k, 0}, i, k=1 ; 2 ; 3$ defines the spatial density of the spin vector. In virtue of (85), we have:

$$
S^{i k, 0}=\frac{1}{4} \bar{\psi}\left\{\gamma^{0} \sigma^{i k}+\sigma^{i k} \gamma^{0}\right\} \psi=\frac{1}{2} \bar{\psi} \gamma^{0} \sigma^{i k} \psi .
$$

It follows from (86) that:

$$
\begin{gathered}
S^{23,0}=\left[V_{1}^{*} V_{2}+V_{2}^{*} V_{1}+V_{3}^{*} V_{4}+V_{4}^{*} V_{3}\right] \mathrm{e}^{-\alpha-2 \beta-\gamma} \\
S^{31,0}=\left[V_{1}^{*} V_{2}-V_{2}^{*} V_{1}+V_{3}^{*} V_{4}-V_{4}^{*} V_{3}\right] \mathrm{e}^{-2 \alpha-\beta-\gamma} \\
S^{12,0}=\left[V_{1}^{*} V_{1}-V_{2}^{*} V_{2}+V_{3}^{*} V_{3}+V_{4}^{*} V_{4}\right] \mathrm{e}^{-2 \alpha-\beta-\gamma} \\
S^{12,0}=S^{13,0}=0, \\
S^{23,0}=\frac{3}{2} a^{2} \mathrm{e}^{-\alpha(\xi)} \cosh [2 N(\xi)]
\end{gathered}
$$

The chronometricaly invariant spatial density of the spinor vector is:

$$
S_{c h l}^{23,0}=\left(S_{23,0} S^{23,0}\right)^{\frac{1}{2}}=\frac{3}{2} a^{2} \cosh [2 \sigma(x)] \mathrm{e}^{-\alpha}=\frac{1}{2} \rho(x)
$$

The projection of the spin vector on the $\xi$ axis is given by the following expression:

$$
S_{1}=\int_{0}^{\xi_{c}} S_{c h I}^{23,0} \sqrt{3-g} \mathrm{~d} x
$$

According to expressions (84) and (93), the total charge $Q$ and the total spin $S_{1}$ are finite.

In the following section, we shall examine the Einstein's, spinor and scalar fields equations by choosing a concrete form of the arbitrary function $F(S)$.

\section{Discussion}

In this section, we shall analyze the general results obtained in the previous sec- 
tion for concrete form of the function $F(S)$. The function $F(S)$ is chosen under the form:

$$
F(S)=\frac{\lambda S^{n}}{1-\lambda S^{n}}
$$

THus, the expression of the function $Q(S)$ becomes:

$$
Q(S)=\frac{1}{1+F(S)}=1-\lambda S^{n} .
$$

By substituting (95) into (51), without losing the generality we can consider massless spinor and scalar fields, according to the theory of Heisenberg [17], we obtain:

$$
S(\xi)=\left\{\frac{C_{1} \sqrt{1+\chi C^{2}}}{C \sqrt{\chi} \cosh \left[n(4+3 B) \sqrt{\frac{1+\chi C^{2}}{3 B^{2}+8 B+4}}\left(\xi+\xi_{0}\right)\right]}\right\}^{\frac{1}{n}}
$$

The function $S=\bar{\psi} \psi$ is a continuous and limited function.

Introducing the relations (94) and (96) into (22), we define the energy density of the interaction of spinor and scalar fields:

$$
\begin{gathered}
T_{0}^{0}(\xi)=\frac{C^{2} S^{2}}{2 C_{1}^{2}[1+F(S)]^{2}}\left[1+F(S)-S \frac{\mathrm{d} F(S)}{\mathrm{d} S}\right] \\
=\frac{C^{2}}{2 C_{1}^{2}}\left\{\frac{C_{1} \sqrt{1+\chi C^{2}}}{C \sqrt{\chi} \cosh \left[n(4+3 B) \sqrt{\frac{1+\chi C^{2}}{3 B^{2}+8 B+4}}\left(\xi+\xi_{0}\right)\right]}\right]^{\frac{2}{n}} \cdot v(\xi),
\end{gathered}
$$

where the function $v(\xi)$ is defined by the following expression:

$$
v(\xi)=\left[1-(n+1)\left\{\frac{C_{1} \sqrt{1+\chi C^{2}}}{C \sqrt{\chi} \cosh \left[n(4+3 B) \sqrt{\frac{1+\chi C^{2}}{3 B^{2}+8 B+4}}\left(\xi+\xi_{0}\right)\right]}\right\}\right]
$$

The distribution of the energy density per unit invariant volume is given by the expression:

$$
f(\xi)=\frac{C^{2}}{2 C_{1}^{2}}\left\{\frac{C_{1} \sqrt{1+\chi C^{2}}}{C \sqrt{\chi} \cosh \left[n(4+3 B) \sqrt{\frac{1+\chi C^{2}}{3 B^{2}+8 B+4}}\left(\xi+\xi_{0}\right)\right]}\right\}^{\frac{2}{n}} \cdot v(\xi) \sin \theta
$$

From (98) and (100), the energy density and the distribution of the energy 
density per unit invariant volume are continuous and localized functions. Moreover, the total energy $E=\int_{0}^{\xi_{c}} T_{0}^{0}(\xi) \sqrt{3_{-g}} \mathrm{~d} \xi$ is limited. The solutions of Equation (31) are soliton-like when the arbitrary function is chosen under the form $F(S)=\frac{\lambda S^{n}}{1-\lambda S^{n}}$.

Let us emphasize that when the interaction lagangian is null i.e the invariant function is null in the relation (1), the obtained solutions are exact and regular. Nevertheless, the energy density is not localized. The lagrangian interaction is very important in order to obtain the regular solutions with localized energy density.

In order to clarify the role of the nonlinear terms $L_{\text {int }}$ of the interaction of spinor, scalar and gravitational fields in the nonlinear fields equations in the formation of regular localized soliton-like solutions, we obtained the soslutions to the fields equations in the case where $L_{i n t}=0$. Let us note that the obtained solutions are exact regular but their energy density is not bounded. In the present case soliton-like solutions are absent.

\section{Concluding Remarks}

Taking into account the proper gravitational field of elementary particles, we have obtained and examined the exact general solutions of interaction scalar, spinor and gravitational field equations. These solutions describe the configurations of scalar and spinor fields with a localized energy density $T_{0}^{0}$, finite total energy $E$. In addition, the total charge and spin are limited.

The solitons-like solutions with limited total spin and charge exist in the case where the arbitrary function $F(S)$ is under the general form $F(S)=\frac{\lambda S^{n}}{1-\lambda S^{n}}$.

\section{Conflicts of Interest}

The authors declare no conflicts of interest regarding the publication of this paper.

\section{References}

[1] Shikin, G.N. (1995) Theory of Solitons in General Relativity. URSS, Moscow.

[2] Adomou, A. and Shikin, G.N. (1998) Exact Self-Consistent Plane-Symmetric Solutions of the Equations of Two Interacting Fields (Spinor Field And Scalar Field). Russian Physics Journal, 41, 672-678. https://doi.org/10.1007/BF02766486

[3] Adomou, A., Alvarado, R. and Shikin, G.N. (1995) Nonlinear Spinor Field Equations in Gravitational Theory. Izvestiya Vuzov, Fizika, 8, 63-68.

[4] Adomou, A., Edou, J. and Massou, S. (2019) plane Symmetric Solutions to the Nonlinear Spinor Field Equations in General Relativity Theory. Journal of Modern Physics, 10, 1222-1234. https://doi.org/10.4236/jmp.2019.1010081

[5] Adomou, A. and Shikin, G.N. (1997) Intearcting Spinor and Scalar Fields: Exact Self-Consistent Plane-Symmetric Solutions. 15th International Conference of General Relativity and gravitation, 16-21 December 1997, Pune. 
[6] Saha, B. and Shikin, G.N. (2001) Nonlinear Spinor and Scalar Fields in General Relativity.

[7] Saha, B. and Shikin, G.N. (2003) Plane Symmetric Solitons of Spinor and Scalar Fields. Czechoslovak Journal of Physics, 54, 597-620.

https://doi.org/10.1023/B:CJOP.0000029690.61308.a5

[8] Adanhoumè, A., Adomou, A., Codo, F.P. and Hounkonnou, M.N. (2012) Nonlinear Spinor Field Equations in Gravitaional Theory: Spherical Symmetric Soliton-Like Solutions. Journal of Modern Physics, 3, 935-942.

https://doi.org/10.4236/jmp.2012.39122

[9] Adomou, A., Edou, J. and Massou, S. (2019) Soliton-Like Spherical Symmetric Solutions of the Nonlinear Spinor Field Equations Depending on the Invariant Function IP = P2 in the General Relativity Theory. Journal of Applied Mathematics and Physics, 7, 2818-2835.

[10] Massou, S., Adomou, A. and Edou, J. (2019) Soliton-Like Spherical Symmetric Solutions of the Nonlinear Spinor Field Equations in General Relativity. International Journal of Applied Mathematics and Theoretical Physics, 5, 118-128. https://doi.org/10.11648/j.ijamtp.20190504.14

[11] Adomou, A., Edou, J., Hontinfinde S.I.V. and Massou, S. (2020) Exact Soliton-Like Spherical Symmetric Solutions of Heisenberg-Ivanenko Nonlinear Spinor Field Equation in Gravitational Theory. Journal of Applied Mathematics and Physics, 8 , 1236-1254. https://doi.org/10.4236/jamp.2020.87094

[12] Adomou, A., Edou, J., Hontinfinde S.I.V. and Massou, S. (2020) Spherical Symmetric Solitons of Spinor Field in Gravitational Theory. International Journal of Advanced Research, 8, 1331-1340.

[13] Edou, J., Adomou, A., Hontinfinde, S.I.V. and Massou, S. (2020) Analytical Soliton-Like Solutions to Nonlinear DIRAC Equations of Spinor Field in Spherical Symmetric Metric. Journal of High Energy Physics, Gravitation and Cosmology, 6, 623-639. https://doi.org/10.4236/jhepgc.2020.64042

[14] Zhelnorovich, V.A. (1982) Theory of Spinors and Its Applications to Physics and Mechanics. Nauka, Moscow.

[15] Brill, D. and Wheeler, J. (1957) Interaction of Neutrinos and Gravitational Fields. Reviews of Modern Physics, 29, 465. https://doi.org/10.1103/RevModPhys.29.465

[16] Bogoliubov, N.N. and Shirkov, D.V. (1976) Introduction to the Theory of Quantized Fields. Nauka, Moscow.

[17] Heisenberg, W. (1966) Introduction to Unified Field Theory of Elementary Particles. Interscience Publishers, London. 